\title{
Exercise reverses the effects of early life stress on orexin cell reactivity in male but not female rats
}

\author{
Morgan H. James ${ }^{1+}$, Erin J. Campbell ${ }^{1+}$, Frederick R. Walker ${ }^{1}$, Doug W. Smith ${ }^{1}$, \\ Heather N. Richardson ${ }^{2}$, Deborah M. Hodgson ${ }^{1}$ and Christopher V. Dayas ${ }^{1 *}$ \\ Neurobiology of Addiction Laboratory, The Centre for Brain and Mental Health Research, School of Biomedical Sciences and Pharmacy, Hunter Medical Research \\ Institute, University of Newcastle, Newcastle, NSW, Australia \\ ${ }^{2}$ Neurobiology of Stress and Addiction Laboratory, Department of Psychology, University of Massachusetts, Amherst, MA, USA
}

Edited by:

Francesca Cirulli, Istituto Superiore di Sanità, Italy

Reviewed by:

Shane M O'Mara, Trinity College

Dublin, Ireland

Deborah Suchecki, Universidade

Federal de São Paulo, Brazil

\section{*Correspondence:}

Christopher V. Dayas, School of

Biomedical Sciences and Pharmacy,

Hunter Medical Research Institute,

Room 306, Medical Sciences

Building, University Drive,

Callaghan, Newcastle, NSW 2308

Australia

e-mail: christopher.dayas@

newcastle.edu.au

${ }^{\dagger}$ These authors have contributed equally to this work.
Early life stress (ELS) is a known antecedent for the development of mood disorders such as depression. Orexin neurons drive arousal and motivated behaviors in response to stress. We tested the hypothesis that ELS alters orexin system function and leads to an altered stress-induced behavioral phenotype in adulthood. We also investigated if voluntary exercise during adolescent development could reverse the ELS-induced changes. Male and female Wistar rats were subjected to maternal separation stress on postnatal days (PND) 2-14. A subset of animals was given access to running wheels in late adolescence (1 hr/day, PND40-70). In adulthood, rats were exposed to restraint stress and then tested on the open field (OF) and elevated plus maze (EPM). Brains were processed for Fos-protein and orexin or tyrosine hydroxylase immunohistochemistry. Restraint stress stimulated Fos-protein expression in perifornical area orexin cells, the paraventricular hypothalamic nucleus, and paraventricular thalamic nuclei, but this neuronal response was dampened in male and female rats exposed to ELS. ELS also reduced exploration in the OF, without affecting EPM behavior. These neural and behavioral changes are consistent with a depressive-like phenotype. Adolescent exercise reversed the orexin and behavioral deficits in ELS males. Exercise was not protective in females, although this may be due to sex differences in running behavior. Our findings highlight the inherent plasticity of the orexin system - a trait that may lead to a state of pathological rewiring but could also be treated using non-pharmacological approaches. We also highlight a need to better understand the sex-specific changes in orexin circuits and stress-related pathology.

Keywords: orexin, hypocretin, stress, maternal separation, sex-differences, exercise, plasticity, hypothalamus

\section{INTRODUCTION}

Early life stress (ELS) is a major risk factor for the emergence of mood-related disorders such as depression and anxiety in adulthood (Danese et al., 2008). Preclinical studies show that separation of rat pups from their mother during the neonatal period (known as maternal separation) also increases vulnerability to anxiety- and depression-like behavior in adulthood (Winslow and Insel, 1991). The impact of ELS on the brain is dramatic and includes maladaptations to the neuroendocrine hypothalamus (i.e., the paraventricular nucleus; PVN) and associated feedback circuits (Meaney et al., 1996, 2007). Importantly, other hypothalamic systems are known to influence autonomic, neuroendocrine, and behavioral responses to stress, but there have been few studies addressing the impact of ELS on these nonneuroendocrine cell groups. For example, cell groups within the lateral hypothalamus (LH) have the capacity to influence a number of stress-relevant behavioral adaptations, including changes in arousal and reward status (Harris and Aston-Jones, 2006; Furlong et al., 2009). Dysregulation of these LH systems by ELS could significantly increase the risk for development of anxiety and depression in later life.
Of particular interest in this context are the orexin (hypocretin) neurons that are now known to be central to LH-mediated changes in arousal and motivational states (Harris and AstonJones, 2006; James et al., 2011, 2012; Johnson et al., 2012). Acute stress robustly increases activation of orexin neurons (Ida et al., 2000; Furlong et al., 2009), whereas chronic stress appears to have an opposite effect (Lutter et al., 2008; Nocjar et al., 2012). The ability of chronic stress to restrict orexin activity is particularly interesting, as evidence has recently emerged linking low orexin system function with depressive symptoms in humans (Brundin et al., 2007, 2009). Surprisingly, the effect of ELS on orexin neuron function in adulthood has not been directly tested. Therefore, the primary aim of this study was to investigate the effects of ELS on orexin system function following psychological stress exposure in adulthood.

Non-pharmacological approaches to produce or augment antidepressant/anxiolytic action have significant clinical relevance and appeal. Both clinical and preclinical studies suggest that physical activity or exercise can produce antidepressant-like effects (Greenwood et al., 2003; Lapmanee et al., 2013). At present however, it is unclear whether the antidepressant or anxiolytic 
effects of physical activity might be linked to improvement in LH-orexin system function. Thus, a secondary aim of the present study was to investigate the possible preventative effects of physical activity on ELS-induced maladaptive orexin cell responses to stress in adulthood. Finally, because very few studies have examined the sex-specific effects of exercise on stress-related behavior, we carried out our experiments in both male and female rats.

\section{METHODS AND MATERIALS ETHICS STATEMENT}

All procedures performed were approved by the University of Newcastle Animal Care and Ethics Committee, and were carried out in accordance with the New South Wales Animal Research Act.

\section{ANIMALS}

Ten experimentally naïve Wistar dams were obtained from the University of Newcastle Animal house and bred with two experimentally naïve males in the University of Newcastle vivarium. A total of 34 male and 39 female offspring were included in the study. As per previous studies (Caldji et al., 2000; Weaver et al., 2007; Nakamura et al., 2011), litters were not standardized to a fixed number of pups or male/female ratio; rather, these variables were accounted for during data analysis (see Data Analysis section below). On postnatal day 1 (PND1), animals from each litter were randomly allocated to the ELS or control (no ELS) condition. ELS allocated litters underwent maternal separation procedures (detailed below) between PND2-14. On PND21, animals were weaned and separated into same-sex housing, with 2 animals/cage $(41.5 \times 28 \times 22 \mathrm{~cm}$ cages; Mascot Wire Works, Sydney). Food (Rat and Mouse Pellets, Glen Forest, Western Australia) and water were available ad libitum and rats were maintained on a 12-h light (0600-1800): $12 \mathrm{~h}$ dark cycle. Temperature was maintained at $20 \pm 2{ }^{\circ} \mathrm{C}$ and humidity was kept at $34 \pm 2 \%$.

\section{EARLY LIFE STRESS}

An overview of the experimental design is outlined in Figure 1. The maternal separation procedure was performed as per previously published procedures in our laboratory (Nakamura et al., 2011), that were based on earlier studies (Plotsky and Meaney, 1993). Briefly, from PND2-14, litters in the ELS condition were removed from their home cage and individually placed in clear separation containers $(13 \times 13 \times 7 \mathrm{~cm})$ in an alternate temperature controlled room $\left(30-34^{\circ} \mathrm{C}\right)$ for $3 \mathrm{~h}$ each day, from 0900 to $1200 \mathrm{~h}$. Pups in the control condition remained undisturbed during this period except for weekly weighing. Bedding was left undisturbed for one week after birth, after which it was changed on a weekly basis.

\section{EXERCISE}

A subgroup of animals exposed to ELS (males $n=6$; females $n=9$ ) was allowed access to a running wheel located in a separate room between PND40-70 $(85 \times 7.5 \mathrm{~cm}, 94 \times 12 \mathrm{~cm}$; Transoniq; for $1 \mathrm{hr} /$ day, 5 days/week between 1800 and $2100 \mathrm{~h}$ ). Only animals exposed to ELS were given access to exercise wheels, as pilot studies indicated that wheel running had no behavioral consequences for animals not exposed to ELS. (i.e., ELS+exercise group did not differ significantly from no-ELS+exercise; see Supplementary Material 1). A rotation counter attached to each wheel quantified distance traveled. Food intake was estimated across all groups during the exercise period by weighing food daily and dividing the change in food weight by the number of animals per cage.

\section{ADULT STRESS EXPOSURE}

Pilot studies revealed that maternal separation had no effect on open field (OF) behavior in the absence of an additional stressor in adulthood (see Supplementary Material 2). As such, between PND75-79, all animals were exposed to $30 \mathrm{~min}$ restraint stress prior to behavioral testing. Animals were removed from their home cage and were placed inside a soft wire mesh restrainer $(25 \times 20 \mathrm{~cm})$ that was folded around the animal and secured with butterfly clips. This procedure has been previously demonstrated to produce a pattern of Fos-activity centered on amygdaloid and brainstem catecholamine nuclei that is distinct from physical stressors (Dayas et al., 1999). Females were tested only in the diestrous phase, monitored using a rat vaginal impedance device (Muromachi Kikai, Tokyo), as described elsewhere (Walker et al., 2010).

\section{BEHAVIORAL TESTING}

Both OF and EPM testing was conducted in darkness using infrared lighting. Time and event data for both apparatuses was recorded using a computer-automated behavioral tracking system (Motion Mensura Ltd., Australia). Immediately following restraint stress, animals were placed in a square $1 \times 1 \mathrm{~m}$ open field task apparatus enclosed by $40 \mathrm{~cm}$ high walls for $10 \mathrm{~min}$. Exploratory variables measured were total distance traveled and time in immobility. Immediately following OF testing, approximately half of the animals (males $n=18$; females $n=24$ ) were tested on the EPM apparatus whilst the remaining animals (males $n=16$; females $n=15$ ) were returned to their home cage. Animals were transferred to a separate room where they were placed on an EPM apparatus. The EPM was painted black, and consisted of two open and two closed arms $(45 \mathrm{~cm}$ length $\times 10 \mathrm{~cm}$ width) as well as a central square $(10 \times 10 \mathrm{~cm})$. The primary measures on this assay included the time spent in the open arms,

\begin{tabular}{|c|c|c|c|c|}
\hline PND 2-14 & PND 40-70 & & PND 75-79 & \\
\hline $\begin{array}{l}\text { Maternal } \\
\text { Separation } \\
\text { 3hrs/day }\end{array}$ & $\begin{array}{c}\text { Running Wheel Access/ } \\
\text { No Access } \\
1 \mathrm{hr} / \text { day }\end{array}$ & $\begin{array}{l}\text { Restraint } \\
\text { 30mins }\end{array}$ & Behavioural Testing $\longrightarrow$ Sacrifice & Immunohistochemistry \\
\hline
\end{tabular}

FIGURE 1 | A schematic illustration of the experimental design. Neonatal treatment consisted of either early life stress (maternal separation) for 3hrs/day from postnatal days (PND) 2-14, or no early life stress. A subgroup of animals was given access to running wheels for $1 \mathrm{hr} /$ day, 5days/week from PND40-70.
All animals were subjected to restraint stress in adulthood (PND75-79) for $30 \mathrm{~min}$. Immediately following restraint, animals underwent behavioral testing in the open field test $(10 \mathrm{~min})$ and elevated plus maze $(5 \mathrm{~min})$. Ninety minutes following restraint stress, animals were euthanized and brains collected. 
an index of anxiety-related behavior, and the number of closed arm entries, a measure of overall locomotor activity (Richardson et al., 2006). We also measured number of entries into the open arms and center square, as well as latency to enter the open and closed arms. Importantly, EPM-challenged animals did not differ from non-EPM-challenged animals in terms of Fos-protein expression in any of the regions studied, and therefore data from these animals were combined.

\section{BRAIN TISSUE HARVESTING AND IMMUNOHISTOCHEMISTRY}

Two hours following the initiation of restraint stress $(1 \mathrm{hr}$ 20min following OF; $1 \mathrm{hr} 15 \mathrm{~min}$ following EPM), rats were deeply anesthetized with sodium pentobarbitone $(200 \mathrm{mg} / \mathrm{kg}$; i.p.; Virbac, Australia). Animals were then perfused with $200 \mathrm{~mL}$ of $0.1 \mathrm{M}$ Phosphate Buffered Saline followed by $500 \mathrm{~mL}$ of $4 \%$ paraformaldehyde ( $\mathrm{pH}$ 9.5). Brains were removed and postfixed in $4 \%$ paraformaldehyde at $4^{\circ} \mathrm{C}$ overnight and then stored in $12.5 \%$ sucrose until sectioning. Serial rostral forebrain $(40-\mu \mathrm{m})$ and caudal midbrain $(50-\mu \mathrm{m})$ sections were cut using a freezing microtome (Leica Microsystems, SM2000R) and a 1-in-4 series of all sections were processed for immunohistochemical detection of Fos-protein (72 h, 1:5000, rabbit polyclonal, Santa Cruz Biotechnology, CA, USA) as described previously in detail (Smith and Day, 1993; Dayas et al., 2008). Hypothalamic sections were dual-labeled for orexin A (48 h, 1:15000, Orexin A antibody, goat polyclonal, Santa Cruz Biotechnology) or in the case of ventral tegmental area (VTA) sections, tyrosine hydroxylase (TH; $48 \mathrm{~h}$, 1:10000, TH antibody, mouse polyclonal, Millipore). An equal number of animals from each treatment group were included in each individual immunohistochemistry run.

Bilateral counts of single-labeled Fos-positive cells were made in the perifornical area (PFA) and lateral hypothalamus (LH; bregma -2.28 to -3.24 ), paraventricular thalamus (PVT; -2.76 to -3.24 ) and medial parvocellular PVN (mpPVN; -1.46 to -1.94). Fos-only cell counts in the PVN and PVT were quantified using Metamorph Imaging System Software (Version 7.5; Molecular Devices Analytical Technologies) at $10 \times$ total magnification (Olympus CX40). The number of Fos-positive cells was determined by creating a region of interest around each structure and a thresholding procedure was used to quantify Fos expression. Counts of Fos-positive orexin neurons in the LH and Fos-positive TH cells in the VTA $(-5.30$ to -5.94$)$ were made by one observer blind to treatment using a $20 \times$ objective (Olympus CX40). In the LH, cell counts were made in the PFA and the LH divisions, as these sections have previously been shown to contain the highest concentration of orexin neurons (Dayas et al., 2008). The PFA was defined as the area surrounding the fornix and the LH was defined as the area from the lateral side of the PFA to the optic tract (Laorden et al., 2012). Cells in the VTA were quantified in the parabrachial pigmented nucleus (PBP) region of the VTA. All brain coordinates were based on the Paxinos and Watson atlas (Paxinos and Watson, 2007).

\section{DATA ANALYSIS}

All statistical analyses were conducted using IBM SPSS V19. Male and female animals were analyzed separately. ANCOVA revealed no significant effect of litter size and male to female ratio for all comparisons. Body weight of treatment groups was compared on PND72 using a one-way between-subjects ANOVA. Food intake and behavioral data were compared across treatment groups using a one-way between-subjects ANOVA and subsequent least significant differences (LSD) post-hoc analyses where appropriate. For immunohistochemical analyses, all cell counts were averaged across each animal for each rostrocaudal level of each brain region examined. To minimize the effects of variability across multiple immunohistochemistry runs, counts for each treatment group were calculated as a fold change relative to control animals processed in the same run. These fold changes were averaged across the rostral-caudal extent of each brain region and were compared across groups using one-way ANOVAs. These analyses were followed by LSD post-hoc analyses where appropriate. An alpha value of 0.05 was adopted for all statistical tests. All figures depict means and standard errors.

\section{RESULTS}

\section{EFFECT OF ELS ON BODY WEIGHT AND FOOD INTAKE}

On PND72, male animals from each treatment group did not differ significantly in terms of their body weight $\left[F_{(2,31)}=\right.$ 2.366, $p=0.106]$, or food intake across the experimental period $\left[F_{(2,14)}=2.554, p=0.113\right]$. Similarly, body weight of females was indistinguishable between treatment groups $\left[F_{(2,36)}=0.026, p=0.975\right]$ as was their food intake $\left[F_{(2,20)}=\right.$ $0.302, p=0.743]$. Interestingly, wheel rotations were on average approximately three times higher in females than male animals in each exercise session $\left[F_{(1,13)}=19.429, p<0.001\right.$; Figure 2].

\section{ELS WAS ASSOCIATED WITH A REDUCED PERCENTAGE OF FOS-POSITIVE OREXIN CELLS AFTER PSYCHOLOGICAL STRESS: PROTECTIVE EFFECT OF EXERCISE ONLY IN MALES}

In male rats there was no effect of treatment on the number of orexin immunoreactive cells in either the PFA or LH subdivisions of the hypothalamus $\left[F_{(2,18)}=0.292, p=0.750 ; F_{(2,18)}=\right.$ $1.648, p=0.220$ respectively, data not shown]. To assess the effect of ELS on the reactivity of orexin neurons to stress in adulthood, we quantified the percentage of orexin cells expressing Fos-protein following psychological stress. ANOVA revealed a significant effect of treatment on the percentage of orexin cells expressing Fos protein in the PFA $\left[F_{(2,18)}=17.646, p<0.001\right]$, and a trend toward significance in the $\operatorname{LH}\left[F_{(2,18)}=3.248, p=\right.$ 0.062]. Post-hoc analyses revealed that ELS animals displayed a significantly lower percentage of orexin neurons that expressed Fos-protein after psychological stress compared to controls in the PFA $(p=0.002)$. Interestingly, ELS animals given access to running wheels displayed a pattern of Fos/orexin immunoreactivity in the PFA that was significantly greater than that of other treatment groups ( $p=0.042$ compared to controls, $p<0.001$ compared to ELS; Figure 3).

Similar to males, orexin cell numbers did not differ across treatment groups in female rats in both the PFA and $\mathrm{LH}$ $\left[F_{(2,18)}=0.141, p=0.87 ; F_{(2,18)}=0.166, p=0.849\right.$, respectively; data not shown]. There was a significant main effect of treatment on the percentage of orexin cells that displayed Foslike immunoreactivity in response to restraint stress in the PFA $\left[F_{(2,18)}=26.907, p<0.001\right]$ and LH $\left[F_{(2,18)}=14.292, p<\right.$ 

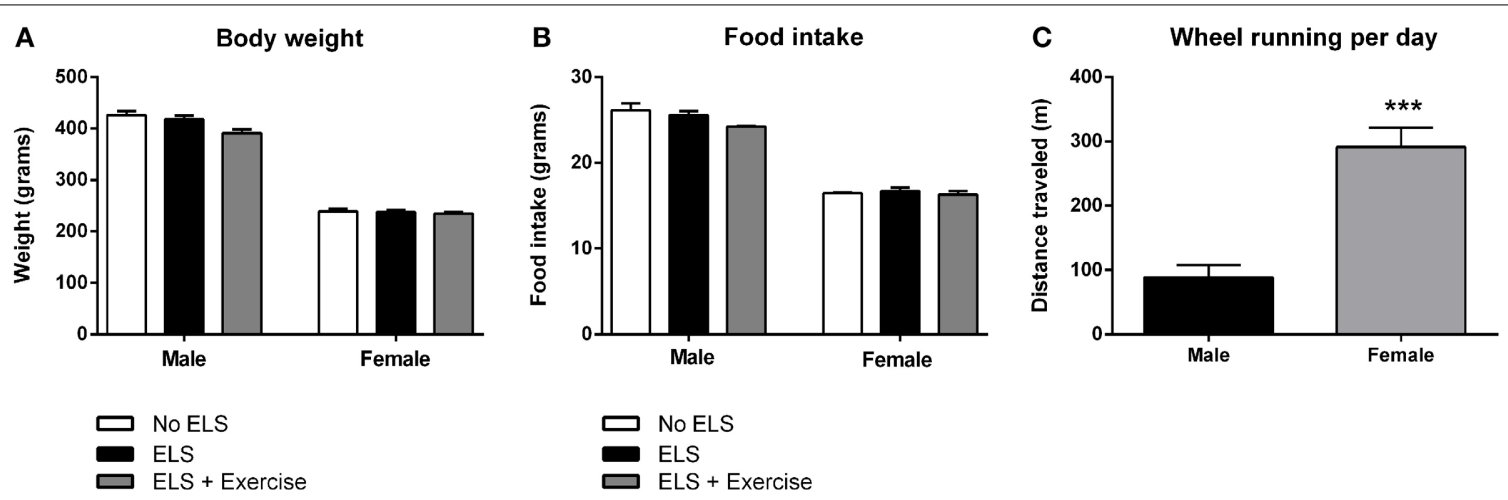

FIGURE 2 | Effect of early life stress (ELS) on body weight and food intake; and sex differences in wheel running. There was no effect of treatment on body weight at postnatal day 72 in both male and female rats. Males: No ELS: $n=13$; ELS: $n=15$; ELS + Ex: $n=6$. Females: No ELS: $n=16$; ELS: $n=14$; ELS + Ex: $n=9$ (A). Similarly, ELS had

no effect on food intake. Males: No ELS: $n=6$; ELS: $n=6$; ELS + Ex $n=5$. Females: No ELS: $n=7$; ELS: $n=7$; ELS + Ex: $n=9$ (B).

Female rats engaged in significantly greater amounts of wheel running per day compared to male rats. Males: $n=6$; Females: $n=9$ (C). *** $p<0.001$.

0.001]. Consistent with male animals, post-hoc analyses showed that ELS females exhibited a significantly lower percentage of Fos-positive orexin cells compared to control animals in the PFA $(p=0.018)$ and a similar trend in the LH $(p=0.094)$. In contrast to males however, access to running wheels tended to exacerbate the effect of treatment on orexin cell reactivity as assessed by Foslabeling in the PFA ( $p<0.001$ compared to controls and ELS) and LH $(p<0.001$ compared to controls, $p<0.01$ compared to ELS; Figure 3).

\section{ELS WAS ASSOCIATED WITH A REDUCTION IN FOS-PROTEIN EXPRESSION IN PVN AND PVT NEURONS FOLLOWING PSYCHOLOGICAL STRESS: PROTECTIVE EFFECT OF EXERCISE IN MALE BUT NOT FEMALE RATS}

In addition to orexin neurons we assessed the level of Fos-like immunoreactivity in the VTA, PVN and PVT following restraint stress in adulthood. In males, the percentage of Fos-positive TH cells in the VTA did not differ significantly between treatment groups $\left[F_{(2,15)}=1.369, p=0.284\right.$; Figure 4]. There was a significant main effect of treatment on Fos-immunoreactivty in the PVN $\left[F_{(2,15)}=9.316, p=0.002\right]$, with post-hoc analyses revealing a significant reduction in Fos-positive cells in ELS animals compared to controls $(p=0.008)$. Access to voluntary exercise significantly increased the number of Fos-positive PVN cells compared to ELS-exposed animals $(p<0.001)$. There was no significant difference between exercised males and controls in this region ( $p=0.287$; Figure 4$)$. In the PVT, there was a significant main effect of treatment on Fos-positive cells $\left[F_{(2,19)}=\right.$ 5.248, $p=0.015]$. Post-hoc analyses revealed a significant increase in Fos-immunoreactivity in the PVT of animals given access to running wheels ( $p=0.023$ compared to controls, and $p=0.006$ compared to ELS; Figure 4). No significant difference was found in the number of Fos-positive cells in the ELS group compared to controls in this region $(p=0.602)$.

In females, there was no significant main effect of treatment on the number of $\mathrm{TH}$-positive cells that expressed Fos-protein $\left[F_{(2,18)}=1.415, p=0.269\right.$; Figure 4]. In the PVN, ANOVA revealed a significant main effect of treatment on the number of
Fos-positive cells $\left[F_{(2,19)}=8.27, p=0.003\right]$. Post-hoc analyses revealed no significant difference between controls and ELS ( $p=$ 0.152 ). However, access to running wheels significantly reduced the number of Fos-positive PVN cells $(p<0.001$ compared to controls, $p=0.016$ compared to ELS; Figure 4). In the PVT, there was a significant main effect of treatment on Fos-protein expression $\left[F_{(2,19)}=6.409, p=0.008\right]$. Post-hoc analyses revealed that there was no significant difference in the number of Fos-positive cells in ELS animals compared to controls $(p=0.156)$. A significant reduction in Fos-positive PVT cells was observed in rats given access to running wheels ( $p=0.002$ compared to controls, $p=0.041$ compared to ELS; Figure 4).

\section{ELS ANIMALS HAD LOWER EXPLORATORY BEHAVIOR IN THE OPEN FIELD FOLLOWING PSYCHOLOGICAL STRESS IN ADULTHOOD: PROTECTIVE EFFECT OF EXERCISE IN MALE BUT NOT FEMALE RATS}

In males, one-way ANOVA revealed a main effect of treatment on the distance traveled in the OF $\left[F_{(2,31)}=2.66, p=0.043\right]$. Post-hoc comparisons revealed that ELS-exposed animals traveled significantly less distance compared to controls $(p=0.026)$. This effect was reversed when ELS animals were given access to voluntary exercise throughout adolescence $(p=0.044$, compared to ELS group; Figure 5). Analyses also showed a trend toward the ELS group exhibiting increased time spent in immobility as compared to the control group, with this effect again being reversed by exercise intervention $\left[F_{(2,31)}=2.21, p=0.063\right.$, data not shown).

In females, there was a significant main effect of treatment in terms of distance traveled $\left[F_{(2,36)}=7.13, p=0.001\right]$. Similar to males, maternally separated animals exhibited locomotor hypoactivity in the OF when compared to controls $(p=0.007)$. In contrast to males, this effect was not reversed by voluntary exercise, but in fact was exaggerated ( $p<0.001$, compared to controls; Figure 5). This same trend was observed in terms of time spent in immobility $\left[F_{(2,36)}=7.44, p=0.001\right]$ with ELS females spending significantly more time in immobility compared to controls $(p=0.01)$. Time spent in immobility was exaggerated in exercised females ( $p<0.001$ compared to controls, data not shown). 


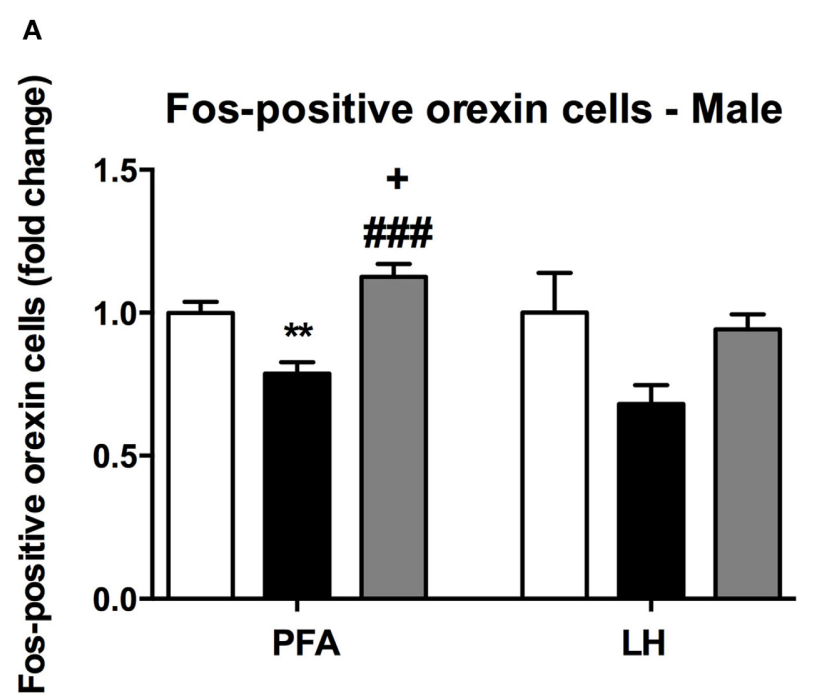

B

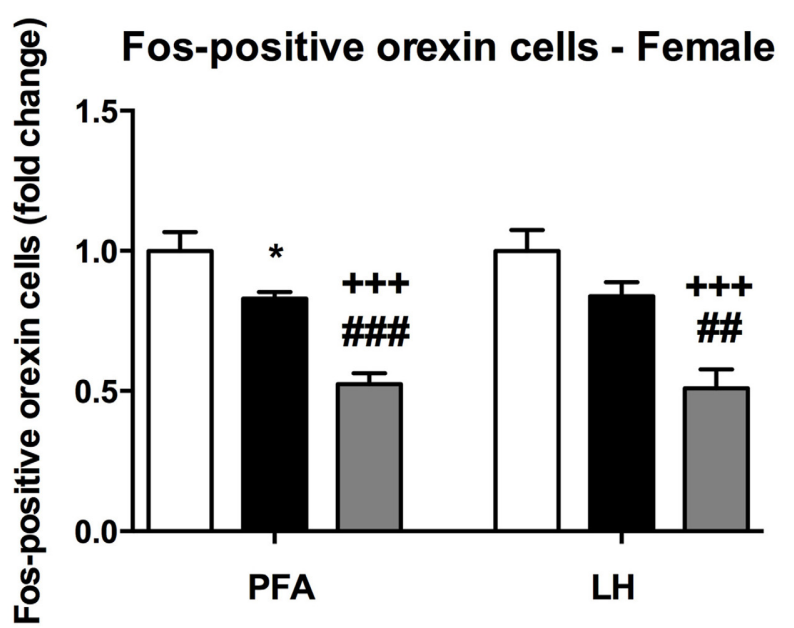

$\square$ No ELS
ELS
ELS + Exercise

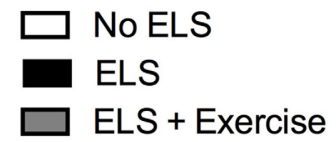

C

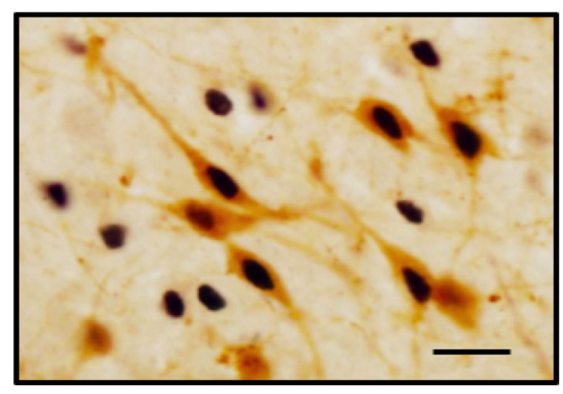

$\mathbf{F}$

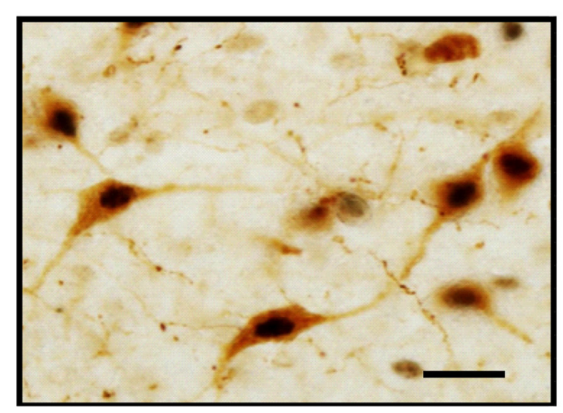

D

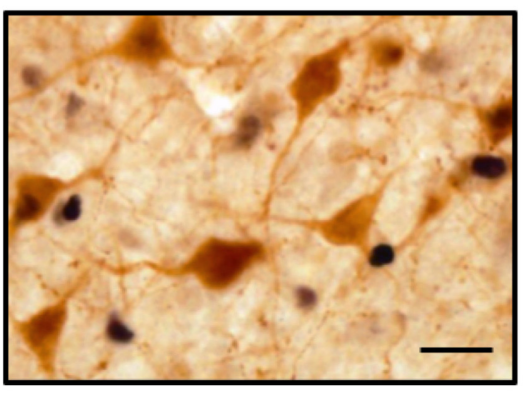

G

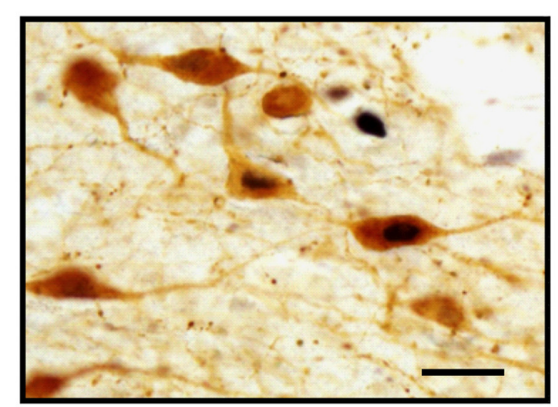

E ELS + Exercise

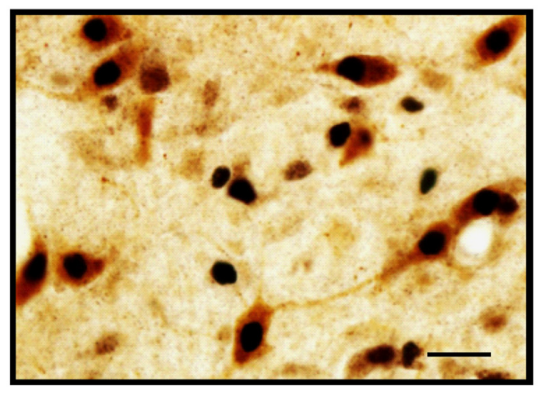

H ELS + Exercise

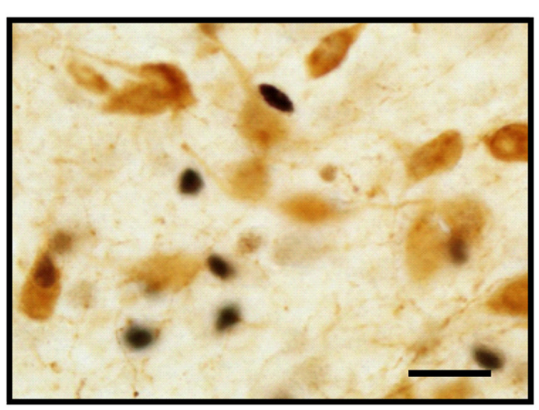

FIGURE 3 | Early life stress (ELS) was associated with a decrease in the percentage of Fos-positive orexin cells in both male and female rats: Wheel running protected against these effects in males, but exaggerated these effects in females. The percentage of Fos-positive orexin cells in the PFA of the hypothalamus was significantly lower in male ELS rats compared to controls. Wheel running was protective against the effects of ELS in this region. This trend was also observed in the $\mathrm{LH}$ but failed to reach significance $(p=0.06 ; \mathbf{A})$. As in male animals, ELS-exposed females exhibited a reduced percentage of Fos-positive orexin cells in the PFA. In contrast to males, wheel running exacerbated these effects in female rats (B). A similar trend was observed in the LH (B). Photomicrographs of coronal sections of the PFA of the hypothalamus immunolabeled for Fos-protein and orexin in males (C,D,E) and females (F,G,H). Males: No ELS: $n=7$; ELS: $n=9$; ELS + Ex: $n=6$. Females: No ELS: $n=7$; ELS: $n=8$, ELS + Ex: $n=7 .{ }^{*} p<0.05$ vs. No ELS, ${ }^{*} p<0.01$ vs. No ELS, ${ }^{+} p<0.05$ vs. No ELS, ${ }^{+++} p<0.001$ vs. No

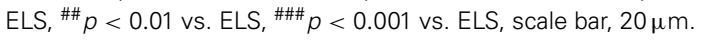



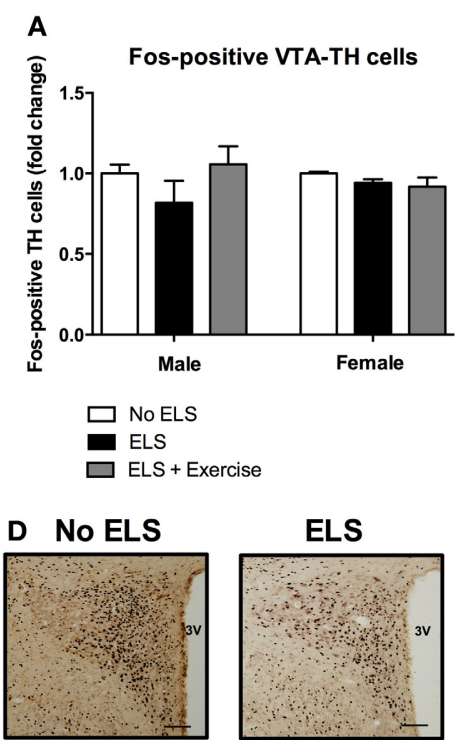

F No ELS

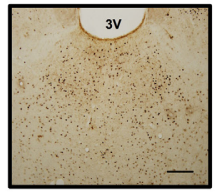

ELS

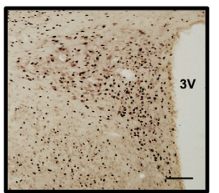

ELS

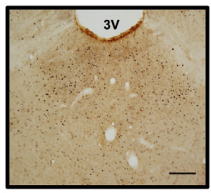

\section{B}

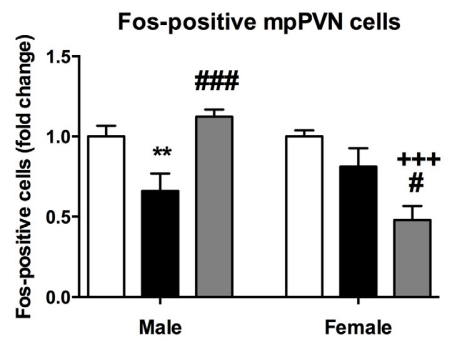

$\square$ NoELS

- ELS

$\square$ ELS + Exercise
C

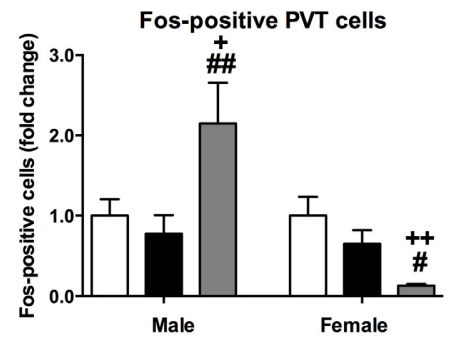

$\square$ NoELS

ELS

$\square$ ELS + Exercise

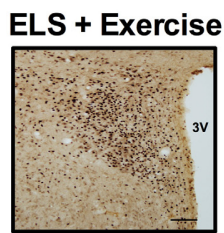

ELS + Exercise

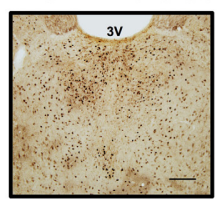

FIGURE 4 | Early life stress (ELS) was associated with a decrease in Fos-immunoreactivity in mpPVN and PVT neurons: Protective effect of exercise in male but not female rats. ELS resulted in a

non-significant reduction in the percentage of Fos-positive TH cells in the VTA in male rats. Wheel running appeared to be protective against these effects. No ELS: $n=6$; ELS: $n=6$, ELS + Ex: $n=6$ (A). In females, there was no effect of ELS or exercise on the percentage of Fos-positive TH cells in the VTA. No ELS: $n=7$; ELS: $n=7$; ELS + Ex: $n=7$ (A). In the PVN, there was an ELS-induced reduction in Fos-positive cells in males and this effect was reversed by wheel running. There was no

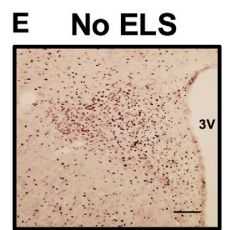

G No ELS

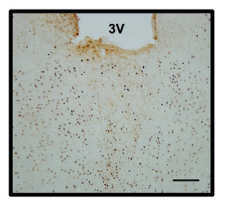

ELS

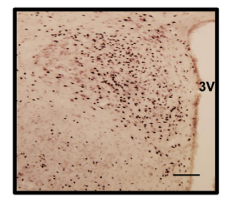

ELS

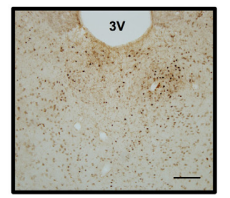

ELS + Exercise

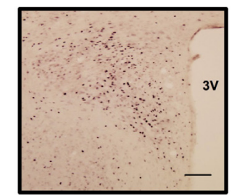

ELS + Exercise

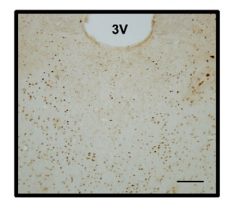

effect of ELS on the number of Fos-positive cells in the PVT in males however, wheel running was protective against the effects of ELS. No ELS: $n=7$; ELS $n=9$; ELS + Ex: $n=6$ (B,C). In females, there was no effect of ELS on the number of Fos-positive cells in the PVN and PVT however, wheel running did exacerbate the effects of ELS. No ELS: $n=7$; ELS: $n=8$; ELS + Ex: $n=7(\mathbf{B}, \mathbf{C})$. Coronal sections of the PVN (males: D; females: E) and PVT (males: $\mathbf{F}$; females: $\mathbf{G}$ ) immunolabeled for Fos-protein, scale bar $100 \mu \mathrm{m}$. ${ }^{* *} p<0.01$ vs. No ELS, $+p<0.05$ vs. No ELS, ${ }^{++} p<0.01$ vs. No ELS, ${ }^{+++} p<0.001$ vs. No ELS, ${ }^{\#} p<0.05$ vs. ELS, \#\# $p<0.01$ vs. ELS, \#\#\# $p<0.001$ vs. ELS.
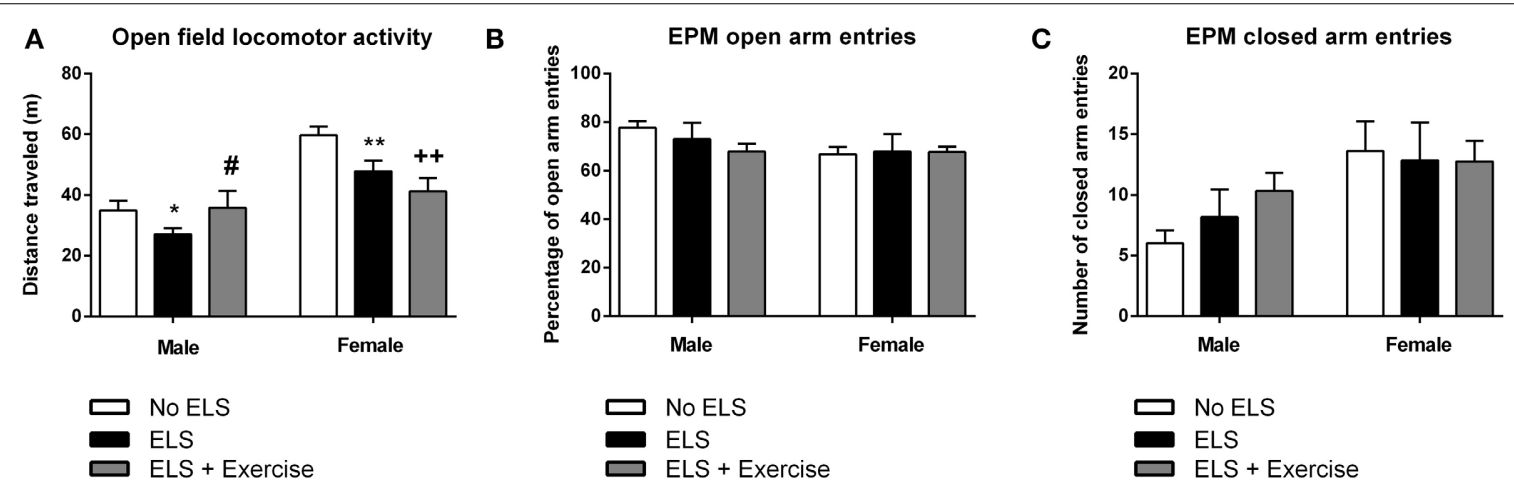

FIGURE 5 | Early life stress (ELS) is associated with reduced locomotor activity in the open field task: Protective effects of exercise in males, but not in females. In both males and females, ELS was associated with a significant reduction in the distance traveled in the open field. Wheel running protected against this effect in male rats, but exacerbated the ELS effect in female rats (A). In both male and female rats, there was no effect of treatment or wheel running on the number of open (B) or closed (C) arm entries across in the elevated plus maze. Males: No ELS: $n=13$; ELS: $n=15$; ELS + Ex: $n=6$. Females: No ELS: $n=16$; ELS: $n=14$; ELS + Ex: $n=9$. ${ }^{*} p<0.05$ vs. No ELS, ${ }^{* *} p<0.01$ vs. No ELS, ${ }^{++} p<0.01$ vs. No ELS, $\# p<0.05$ vs. ELS. 
With respect to the EPM, there was no effect of treatment on the number of open $\left[F_{(2,15)}=0.382, p=0.689\right]$ or closed $\left[F_{(2,15)}=1.624, p=0.230\right]$ arm entries in males. Similarly, in females there was no difference between treatment groups on open $\left[F_{(2,21)}=0.617, p=0.549\right]$ or closed arm entries $\left[F_{(2,21)}=0.040, p=0.961 ;\right.$ Figure 5]. Additionally, there was no effect of treatment on the duration spent in the open arms, closed arms or the center square, or the latency to enter the open or closed arms in either males or females ( $p$ 's $>0.05$ ).

\section{DISCUSSION}

In the present study we report that ELS-exposed male and female rats exhibited a "hypoactive" orexin cell response to restraint stress, as assessed by Fos-like immunoreactivity, particularly in the PFA. Notably, both male and female animals exposed to ELS displayed reduced exploratory behavior on the OF following restraint stress. Interestingly, the ability of exercise to ameliorate ELS-induced deficits was strongly sex-dependent. A similar level of sex-specificity was also seen in brain regions that are known to respond to orexin innervation. Together these results not only highlight the profound effect that ELS has on orexin function in adulthood but also the positive effects of exercise on this deficit and how this differs across sexes.

The primary aim of this study was to assess the impact of ELS on subsequent orexin cell reactivity to psychological stress in adulthood. Due to known sex differences in neural responses to stress, we examined the degree to which orexin cells had become activated by quantifying the number of orexin cells that were Fos-positive following psychological stress in adulthood in both male and female rats. Using this well-characterized strategy, orexin function in both males and females that were exposed to ELS was substantially lower than non-ELS controls. ELS-exposed animals also exhibited significantly lower activity in the OF. These findings are interesting in light of recent findings from other preclinical studies that have shown that chronic stress results in reduced orexin system function and increased depressive-like behavior (Lutter et al., 2008; Nocjar et al., 2012). Further, recent human studies have reported an inverse relationship between CSF orexin peptide levels and symptoms of depression (Brundin et al., 2007, 2009). With these findings in mind, it is possible that in our study, reduced orexin activity induced by ELS resulted in a depressive-like behavioral state that manifested as reduced exploratory behavior. Future studies should assess whether these changes in orexin cell function also manifest as deficits in motivated behavior on behavioral assays such as the sucrose preference test and/or forced swim test. Further investigation is also warranted to understand the relevance of our observation that orexin hypoactivity was more pronounced in the PFA compared to the $\mathrm{LH}$, as separate functions have been ascribed to these populations (stress reactivity and reward-seeking, respectively; Harris and Aston-Jones, 2006).

Perhaps the most striking observation of the present study was that behavioral deficits associated with ELS were not observed in male rats allowed access to voluntary exercise. These findings are consistent with previous studies demonstrating that voluntary wheel running protects against the expression of anxiety-like behavior in adult male rats exposed to maternal separation stress (Maniam and Morris, 2010) or footshock stress in adulthood (Greenwood et al., 2013). Given that there was a significant "wash-out" period between wheel running and restraint, it is likely that exercise reversed ELS-induced changes in LHorexin circuit function rather than prevented the acute effects of restraint. However, we acknowledge further tests are required to address this issue. With respect to the sex-specific effects we observed, our findings are in line with those of Brocardo et al. (2012) who showed that voluntary exercise had no effect on the expression of anxiety- and depression-like behaviors in female rats exposed to ethanol in early life, despite this intervention having protective effects in males (Brocardo et al., 2012). Similarly, findings from the addiction field have yielded differential effects of voluntary exercise on drug-related behaviors in males and females, despite being exposed to identical exercise regimes (Smith et al., 2008; Ehringer et al., 2009; Thanos et al., 2010). Our findings that exercise actually tended to exacerbate ELS-induced orexin and behavioral changes in females perhaps points to the possibility that the increased wheel running observed in females was actually stress provoking. Exercise-induced corticosterone secretion may have subsequently influenced orexin cell responsivity (Ford et al., 2005). These findings point to the need for a greater understanding of how exercise conditions (type, intensity, duration) can be modified to produce beneficial effects in both sexes.

Interestingly, we observed a similar pattern of reactivity in key orexinergic targets, including the mpPVN, PVT, and VTA dopamine neurons. With respect to the mpPVN, there is now considerable evidence that orexin directly modulates the neuroendocrine response to stress. The mpPVN is densely innervated by orexinergic terminals and mainly expresses orexin receptor 2 (Peyron et al., 1998; Trivedi et al., 1998). Further, central administration of orexin-A induces Fos-protein expression in corticotropin releasing factor (CRF)-expressing cells in the mpPVN (Sakamoto et al., 2004) and increases plasma corticosterone and adrenocorticoptropin hormone (ACTH) levels (Ida et al., 2000; Kuru et al., 2000). Whilst we did not directly measure HPA-axis activity, maternal separation stress has previously been shown to be associated with impaired mpPVN and HPAaxis responsivity to stress in adulthood (Plotsky and Meaney, 1993; Ladd et al., 2000; Daniels et al., 2004). Further studies are required to assess whether this impairment is directly associated with the reduction in orexin function observed here. With respect to the PVT, this region is also known to be densely innervated by orexin terminals (Kirouac et al., 2005) and contains high densities of both orexin receptors (Marcus et al., 2001). Orexin signaling in the PVT has recently been shown to be important for both the neuroendocrine response to stress (Heydendael et al., 2012) and the expression of stress-related behaviors (Li et al., 2010a,b; James and Dayas, 2013; Yeoh et al., 2014). Further, stimulation of the PVT can modulate dopamine release in the nucleus accumbens (NAC; Jones et al., 1989; Parsons et al., 2007). Reduced PVT signaling in ELS animals may therefore contribute to reduced striatal dopamine release, an outcome consistent with previous studies showing that hypoactivity of the VTA dopamine-NAC projection is causally linked to depressive-like behavior following social defeat (Berton et al., 2006).

One caveat of our experimental design is that OF and EPM testing were performed after restraint stress. While we attribute 
the pattern of Fos-protein expression observed as a response to restraint stress, given the time course typically required for maximal Fos-protein induction (2 h; Kovács, 1998), we cannot exclude the possibility that OF and EPM testing also influenced the expression of this immediate early gene. Regardless, these challenges are typically regarded as psychological stressors, which produce similar patterns of Fos-protein expression in stress-sensitive brain regions as restraint stress (Dayas et al., 2001). Further, no differences were observed in terms of Fos-protein expression amongst EPM-challenged vs. non-EPM-challenged animals, suggesting that behavioral testing did not have any confounding effects on Fos expression. It is also important to note that a previous report failed to observe an increase in Fos-protein expression in orexin-positive neurons in response to restraint (Furlong et al., 2009). These experiments however, were carried out in animals with no prior stress exposure, and the effects observed in our study may reflect a more important role for orexin signaling in stress reactivity in chronically stressed animals.

In summary, the present study provides novel evidence that the orexin system's response to adult stress is altered by ELS. Identical effects of ELS on orexin cell activity in stressed adults were observed in the PFA of male and female rats. These data are consistent with recent clinical evidence indicating that vulnerability to stress-related mood disorders is linked with orexin system hypofunction (Brundin et al., 2007, 2009). We also show that exercise was protective against both the behavioral (OF activity) and neural effects of ELS in male rats, suggesting that the beneficial effects of exercise on stress-related behavior is associated with a "normalization" of orexin function and that, under some conditions, the orexin system can be modified by non-pharmacological methods. Surprisingly, female rats exhibited significantly greater deficits in orexin function following wheel running, suggesting that while the effects of ELS on orexin function are similar across sexes, future studies will need to consider alternative approaches to recover orexin function in female rats. These findings extend recent studies showing that the orexin system is highly plastic and is readily modified by environmental events (Yeoh et al., 2012). More broadly, this study highlights the importance of studying sex-based differences in stress-related pathology (Clayton and Collins, 2014).

\section{ACKNOWLEDGMENTS}

We would like to acknowledge the support of Dr. Javad Bourei for his assistance with the maternal separation procedures. We would also like to thank Dr. Luba Sominsky, Ms. Emily Levi, Ms. Jessica Buck, and Ms. Sarahanne Field for their assistance with the experiments. This work was supported by grants to Christopher V. Dayas from the Hunter Medical Research Institute (HMRI), the Sparke Helmore/NBN Television Triathlon Festival Grant, the Leslie McFarlane Bequest Grant, and NHMRC Project Grant 510778. This work was also supported by funding to Morgan $\mathrm{H}$. James from NHMRC CJ Martin Fellowship 1072706, HMRI, and PULSE.

\section{SUPPLEMENTARY MATERIAL}

The Supplementary Material for this article can be found online at: http://www.frontiersin.org/journal/10.3389/fnbeh. 2014.00244/abstract

\section{REFERENCES}

Berton, O., McClung, C. A., Dileone, R. J., Krishnan, V., Renthal, W., Russo, S. J., et al. (2006). Essential role of BDNF in the mesolimbic dopamine pathway in social defeat stress. Science 311, 864-868. doi: 10.1126/science.1120972

Brocardo, P. S., Boehme, F., Patten, A., Cox, A., Gil-Mohapel, J., and Christie, B. R. (2012). Anxiety- and depression-like behaviors are accompanied by an increase in oxidative stress in a rat model of fetal alcohol spectrum disorders: Protective effects of voluntary physical exercise. Neuropharmacology 62, 1607-1618. doi: 10.1016/j.neuropharm.2011.10.006

Brundin, L., Bjorkqvist, M., Petersen, A., and Traskman-Bendz, L. (2007). Reduced orexin levels in the cerebrospinal fluid of suicidal patients with major depressive disorder. Eur. Neuropsychopharmacol. 17, 573-579. doi: 10.1016/j.euroneuro.2007.01.005

Brundin, L., Bjorkqvist, M., Traskman-Bendz, L., and Petersen, A. (2009). Increased orexin levels in the cerebrospinal fluid the first year after a suicide attempt. J. Affect. Disord. 113, 179-182. doi: 10.1016/j.jad.2008.04.011

Caldji, C., Francis, D., Sharma, S., Plotsky, P. M., and Meaney, M. J. (2000). The effects of early rearing environment on the development of GABAA and central benzodiazepine receptor levels and novelty-induced fearfulness in the rat. Neuropsychopharmacology 22, 219-229. doi: 10.1016/S0893-133X(99) 00110-4

Clayton, J. A., and Collins, F. S. (2014). Policy: NIH to balance sex in cell and animal studies. Nature 509, 282-283. doi: 10.1038/509282a

Danese, A., Moffitt, T. E., Pariante, C. M., Ambler, A., Poulton, R., and Caspi, A. (2008). Elevated inflammation levels in depressed adults with a history of childhood maltreatment. Arch. Gen. Psychiatry 65, 409-415. doi: 10.1001/archpsyc.65.4.409

Daniels, W. M., Pietersen, C. Y., Carstens, M. E., and Stein, D. J. (2004). Maternal separation in rats leads to anxiety-like behavior and a blunted ACTH response and altered neurotransmitter levels in response to a subsequent stressor. Metab. Brain Dis. 19, 3-14. doi: 10.1023/B:MEBR.0000027412.19664.b3

Dayas, C. V., Buller, K. M., Crane, J. W., Xu, Y., and Day, T. A. (2001). Stressor categorization: acute physical and psychological stressors elicit distinctive recruitment patterns in the amygdala and in medullary noradrenergic cell groups. Eur. J. Neurosci. 14, 1143-1152. doi: 10.1046/j.0953-816x.2001.01733.x

Dayas, C. V., Buller, K. M., and Day, T. A. (1999). Neuroendocrine responses to an emotional stressor: evidence for involvement of the medial but not the central amygdala. Eur. J. Neurosci. 11, 2312-2322. doi: 10.1046/j.14609568.1999.00645.x

Dayas, C. V., Mcgranahan, T. M., Martin-Fardon, R., and Weiss, F. (2008). Stimuli linked to ethanol availability activate hypothalamic CART and orexin neurons in a reinstatement model of relapse. Biol. Psychiatry 63, 152-157. doi: 10.1016/j.biopsych.2007.02.002

Ehringer, M. A., Hoft, N. R., and Zunhammer, M. (2009). Reduced alcohol consumption in mice with access to a running wheel. Alcohol 43, 443-452. doi: 10.1016/j.alcohol.2009.06.003

Ford, G. K., Al-Barazanji, K. A., Wilson, S., Jones, D. N., Harbuz, M. S., and Jessop, D. S. (2005). Orexin expression and function: glucocorticoid manipulation, stress, and feeding studies. Endocrinology 146, 3724-3731. doi: 10.1210/en. 2005-0496

Furlong, T. M., Vianna, D. M. L., Liu, L., and Carrive, P. (2009). Hypocretin/orexin contributes to the expression of some but not all forms of stress and arousal. Eur. J. Neurosci. 30, 1603-1614. doi: 10.1111/j.1460-9568.2009.06952.x

Greenwood, B. N., Foley, T. E., Day, H. E., Campisi, J., Hammack, S. H., Campeau, S., et al. (2003). Freewheel running prevents learned helplessness/behavioral depression: role of dorsal raphe serotonergic neurons. J. Neurosci. 23, 2889-2898.

Greenwood, B. N., Spence, K. G., Crevling, D. M., Clark, P. J., Craig, W. C., and Fleshner, M. (2013). Exercise-induced stress resistance is independent of exercise controllability and the medial prefrontal cortex. Eur. J. Neurosci. 37, 469-478. doi: 10.1111/ejn.12044

Harris, G. C., and Aston-Jones, G. (2006). Arousal and reward: a dichotomy in orexin function. Trends Neurosci. 29, 571-577. doi: 10.1016/j.tins.2006.08.002

Heydendael, W., Sengupta, A., and Bhatnagar, S. (2012). Putative genes mediating the effects of orexins in the posterior paraventricular thalamus on neuroendocrine and behavioral adaptations to repeated stress. Brain Res. Bull 89, 203-210. doi: 10.1016/j.brainresbull.2012.09.002

Ida, T., Nakahara, K., Murakami, T., Hanada, R., Nakazato, M., and Murakami, N. (2000). Possible involvement of orexin in the stress reaction in rats. Biochem. Biophys. Res. Commun. 270, 318-323. doi: 10.1006/bbrc.2000.2412 
James, M. H., Charnley, J. L., Levi, E. M., Jones, E., Yeoh, J. W., Smith, D. W., et al. (2011). Orexin-1 receptor signalling within the ventral tegmental area, but not the paraventricular thalamus, is critical to regulating cue-induced reinstatement of cocaine-seeking. Int. J. Neuropsychopharmacol. 14, 684-690. doi: $10.1017 /$ S1461145711000423

James, M. H., and Dayas, C. V. (2013). What about me...? The PVT: a role for the paraventricular thalamus (PVT) in drug-seeking behavior. Front. Behav. Neurosci. 7:18. doi: 10.3389/fnbeh.2013.00018

James, M. H., Yeoh, J. W., Graham, B. A., and Dayas, C. V. (2012). Insights for developing pharmacological treatments for psychostimulant relapse targeting hypothalamic peptide systems. Addict. Res. Ther. 4, 1-13. doi: 10.4172/21556105.S4-008

Johnson, P. L., Molosh, A., Fitz, S. D., Truitt, W. A., and Shekhar, A. (2012). Orexin, stress, and anxiety/panic states. Prog. Brain Res. 198, 133-161. doi: 10.1016/B978-0-444-59489-1.00009-4

Jones, M. W., Kilpatrick, I. C., and Phillipson, O. T. (1989). Regulation of dopamine function in the nucleus accumbens of the rat by the thalamic paraventricular nucleus and adjacent midline nuclei. Exp. Brain Res. 76, 572-580. doi: 10.1007/BF00248914

Kirouac, G. J., Parsons, M. P., and Li, S. (2005). Orexin (hypocretin) innervation of the paraventricular nucleus of the thalamus. Brain Res. 1059, 179-188. doi: 10.1016/j.brainres.2005.08.035

Kovács, K. J. (1998). Invited review c-Fos as a transcription factor: a stressful (re)view from a functional map. Neurochem. Int. 33, 287-297. doi: 10.1016/S0197-0186(98)00023-0

Kuru, M., Ueta, Y., Serino, R., Nakazato, M., Yamamoto, Y., Shibuya, I., et al. (2000). Centrally administered orexin/hypocretin activates HPA axis in rats. Neuroreport 11, 1977-1980. doi: 10.1097/00001756-200006260-00034

Ladd, C. O., Huot, R. L., Thrivikraman, K. V., Nemeroff, C. B., Meaney, M. J., and Plotsky, P. M. (2000). Long-term behavioral and neuroendocrine adaptations to adverse early experience. Prog. Brain Res. 122, 81-103. doi: 10.1016/S00796123(08)62132-9

Laorden, M. L., Ferenczi, S., Pinter-Kubler, B., Gonzalez-Martin, L. L., Lasheras, M. C., Kovacs, K. J., et al. (2012). Hypothalamic orexin-A neurons are involved in the response of the brain stress system to morphine withdrawal. PLoS ONE 7:e36871. doi: 10.1371/journal.pone.0036871

Lapmanee, S., Charoenphandhu, J., and Charoenphandhu, N. (2013). Beneficial effects of fluoxetine, reboxetine, venlafaxine, and voluntary running exercise in stressed male rats with anxiety- and depression-like behaviors. Behav. Brain Res. 250, 316-325. doi: 10.1016/j.bbr.2013.05.018

Li, Y., Li, S., Wei, C., Wang, H., Sui, N., and Kirouac, G. J. (2010a). Changes in emotional behavior produced by orexin microinjections in the paraventricular nucleus of the thalamus. Pharmacol. Biochem. Behav. 95, 121-128. doi: 10.1016/j.pbb.2009.12.016

Li, Y., Li, S., Wei, C., Wang, H., Sui, N., and Kirouac, G. J. (2010b). Orexins in the paraventricular nucleus of the thalamus mediate anxiety-like responses in rats. Psychopharmacology (Berl). 212, 251-265. doi: 10.1007/s00213-010-1948-y

Lutter, M., Krishnan, V., Russo, S. J., Jung, S., Mcclung, C. A., and Nestler, E. J. (2008). Orexin signaling mediates the antidepressant-like effect of calorie restriction. J. Neurosci. 28, 3071-3075. doi: 10.1523/JNEUROSCI.5584-07.2008

Maniam, J., and Morris, M. J. (2010). Voluntary exercise and palatable high-fat diet both improve behavioural profile and stress responses in male rats exposed to early life stress: role of hippocampus. Psychoneuroendocrinology 35, 1553-1564. doi: 10.1016/j.psyneuen.2010.05.012

Marcus, J. N., Aschkenasi, C. J., Lee, C. E., Chemelli, R. M., Saper, C. B., Yanagisawa, M., et al. (2001). Differential expression of orexin receptors 1 and 2 in the rat brain. J. Comp. Neurol. 435, 6-25. doi: 10.1002/cne.1190

Meaney, M. J., Diorio, J., Francis, D., Widdowson, J., Laplante, P., Caldji, C., et al. (1996). Early environmental regulation of forebrain glucocorticoid receptor gene expression: implications for adrenocortical responses to stress. Dev. Neurosci. 18, 49-72. doi: 10.1159/000111395

Meaney, M. J., Szyf, M., and Seckl, J. R. (2007). Epigenetic mechanisms of perinatal programming of hypothalamic-pituitary-adrenal function and health. Trends Mol. Med. 13, 269-277. doi: 10.1016/j.molmed.2007.05.003

Nakamura, T., Walker, A. K., Sominsky, L., Allen, T., Rosengren, S., and Hodgson, D. M. (2011). Maternal separation in early life impairs tumor immunity in adulthood in the F344 rat. Stress 14, 335-343. doi: 10.3109/10253890.2010. 548014

Nocjar, C., Zhang, J., Feng, P., and Panksepp, J. (2012). The social defeat animal model of depression shows diminished levels of orexin in mesocortical regions of the dopamine system, and of dynorphin and orexin in the hypothalamus. Neuroscience 218, 138-153. doi: 10.1016/j.neuroscience.2012.05.033

Parsons, M. P., Li, S., and Kirouac, G. J. (2007). Functional and anatomical connection between the paraventricular nucleus of the thalamus and dopamine fibers of the nucleus accumbens. J. Comp. Neurol. 500, 1050-1063. doi: $10.1002 /$ cne. 21224

Paxinos, G., and Watson, C. (2007). The Rat Brain in Stereotaxic Coordinates Sydney, NSW: Academic Press.

Peyron, C., Tighe, D. K., Van Den Pol, A. N., De Lecea, L., Heller, H. C., Sutcliffe, J. G., et al. (1998). Neurons containing hypocretin (orexin) project to multiple neuronal systems. J. Neurosci. 18, 9996-10015.

Plotsky, P. M., and Meaney, M. J. (1993). Early, postnatal experience alters hypothalamic corticotropin-releasing factor (CRF) mRNA, median eminence CRF content and stress-induced release in adult rats. Brain Res. Mol. Brain Res. 18, 195-200. doi: 10.1016/0169-328X(93)90189-V

Richardson, H. N., Zorrilla, E. P., Mandyam, C. D., and Rivier, C. L. (2006) Exposure to repetitive versus varied stress during prenatal development generates two distinct anxiogenic and neuroendocrine profiles in adulthood. Endocrinology 147, 2506-2517. doi: 10.1210/en.2005-1054

Sakamoto, F., Yamada, S., and Ueta, Y. (2004). Centrally administered orexin-A activates corticotropin-releasing factor-containing neurons in the hypothalamic paraventricular nucleus and central amygdaloid nucleus of rats: possible involvement of central orexins on stress-activated central CRF neurons. Regul. Pept. 118, 183-191. doi: 10.1016/j.regpep.2003.12.014

Smith, D. W., and Day, T. A. (1993). Neurochemical identification of fos-positive neurons using two-colour immunoperoxidase staining. J. Neurosci. Methods 47, 73-83. doi: 10.1016/0165-0270(93)90023-K

Smith, M. A., Schmidt, K. T., Iordanou, J. C., and Mustroph, M. L. (2008) Aerobic exercise decreases the positive-reinforcing effects of cocaine. Drug Alcohol Depend. 98, 129-135. doi: 10.1016/j.drugalcdep.2008.05.006

Thanos, P. K., Tucci, A., Stamos, J., Robison, L., Wang, G. J., Anderson, B. J., et al. (2010). Chronic forced exercise during adolescence decreases cocaine conditioned place preference in Lewis rats. Behav. Brain Res. 215, 77-82. doi: 10.1016/j.bbr.2010.06.033

Trivedi, P., Yu, H., Macneil, D. J., Van Der Ploeg, L. H., and Guan, X. M. (1998). Distribution of orexin receptor mRNA in the rat brain. FEBS Lett. 438, 71-75. doi: 10.1016/S0014-5793(98)01266-6

Walker, A. K., Nakamura, T., and Hodgson, D. M. (2010). Neonatal lipopolysaccharide exposure alters central cytokine responses to stress in adulthood in Wistar rats. Stress 13, 506-515. doi: 10.3109/10253890.2010.489977

Weaver, S. A., Diorio, J., and Meaney, M. J. (2007). Maternal separation leads to persistent reductions in pain sensitivity in female rats. J. Pain 8, 962-969. doi: 10.1016/j.jpain.2007.07.001

Winslow, J. T., and Insel, T. R. (1991). The infant rat separation paradigm: a novel test for novel anxiolytics. Trends Pharmacol. Sci. 12, 402-404. doi: 10.1016/0165 6147(91)90616-Z

Yeoh, J. W., Campbell, E. J., James, M. H., Graham, B. A., and Dayas, C. V. (2014). Orexin antagonists for neuropsychiatric disease: progress and potential pitfalls. Front. Neurosci. 8:36. doi: 10.3389/fnins.2014.00036

Yeoh, J. W., James, M. H., Jobling, P., Bains, J. S., Graham, B. A., and Dayas, C. V. (2012). Cocaine potentiates excitatory drive in the perifornical/lateral hypothalamus. J. Physiol. 590, 3677-3689. doi: 10.1113/jphysiol.2012.230268

Conflict of Interest Statement: The authors declare that the research was conducted in the absence of any commercial or financial relationships that could be construed as a potential conflict of interest.

Received: 20 March 2014; accepted: 26 June 2014; published online: 23 July 2014. Citation: James MH, Campbell EJ, Walker FR, Smith DW, Richardson HN, Hodgson DM and Dayas CV (2014) Exercise reverses the effects of early life stress on orexin cell reactivity in male but not female rats. Front. Behav. Neurosci. 8:244. doi: 10.3389/ fnbeh.2014.00244

This article was submitted to the journal Frontiers in Behavioral Neuroscience. Copyright (C) 2014 James, Campbell, Walker, Smith, Richardson, Hodgson and Dayas. This is an open-access article distributed under the terms of the Creative Commons Attribution License (CC BY). The use, distribution or reproduction in other forums is permitted, provided the original author(s) or licensor are credited and that the original publication in this journal is cited, in accordance with accepted academic practice. No use, distribution or reproduction is permitted which does not comply with these terms 